\title{
Phase II Study of Irinotecan and Cisplatin Combination Chemotherapy in Metastatic, Unresectable Esophageal Cancer
}

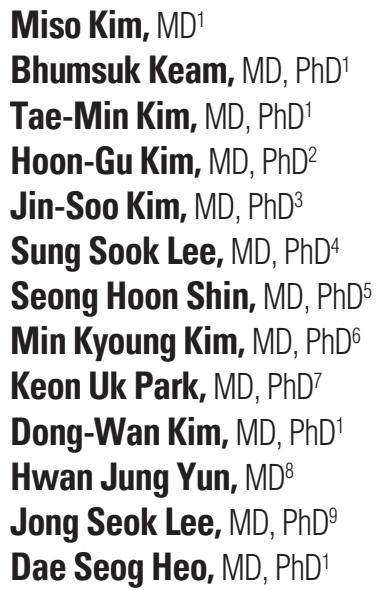

${ }^{*}$ A list author's affiliations appears at the end of the paper.

\section{Purpose}

The objective of this multicenter phase II study was to evaluate the efficacy and safety of irinotecan and cisplatin combination chemotherapy in metastatic, unresectable esophageal cancer.

\section{Materials and Methods}

Patients were treated with irinotecan $65 \mathrm{mg} / \mathrm{m}^{2}$ and cisplatin $30 \mathrm{mg} / \mathrm{m}^{2}$ on days 1 and 8 of each 21-day treatment cycle. The primary endpoint was response rate, and secondary endpoints were survival, duration of response, initial metabolic response rate, and toxicity.

\section{Results}

A total of 27 patients with squamous cell histology were enrolled in the study. The median age of the patients was 61 years. The objective response rate of the 20 patients in the perprotocol group was $30.0 \%$ (90\% confidence interval [Cl], 13.2 to 46.9). The median followup duration was 10.0 months, and the median progression-free survival and overall survival were 4.5 months ( $95 \% \mathrm{Cl}, 1.6$ to 6.2$)$ and 8.8 months ( $95 \% \mathrm{Cl}, 4.7$ to 10.5$)$, respectively. Four of 13 patients (30.8\%) evaluated showed initial metabolic response. The median duration of response for partial responders was 5.0 months (range, 3.4 to 8.0 months). The following grade 3/4 treatment-related hematologic toxicities were reported: neutropenia (40.7\%), anaemia (22.2\%), and thrombocytopenia (7.4\%). Two patients experienced febrile neutropenia. The most common grade 3/4 non-hematologic toxicities were asthenia (14.8\%) and diarrhoea (11.1\%).

\section{Conclusion}

Irinotecan and cisplatin combination chemotherapy showed modest anti-tumour activity and manageable toxicity for patients with metastatic, unresectable esophageal cancer.
Seoul National University Hospital,

Seoul National University College of Medicine,

101 Daehak-ro, Jongno-gu, Seoul 03080, Korea

Tel: 82-2-2072-7215

Fax: 82-2-2072-7379

E-mail: bhumsuk@snu.ac.kr

Received March 18, 2016

Accepted July 13, 2016

Published Online July 28, 2016

\section{Key words}

Esophageal neoplasms, Irinotecan, Cisplatin, Phase II clinical trial

\section{Introduction}

Esophageal cancer is a devastating disease, being the sixth leading cause of cancer deaths worldwide [1] and the eleventh leading cause of cancer deaths in Korea [2]. The incidence of adenocarcinoma in esophageal cancer is gradually increasing in Western countries, but squamous cell carcinoma remains the most predominant histology in Asia. At the time of diagnosis, approximately half of esophageal can- cer patients present with metastatic disease, which is associated with a poor prognosis. The 5-year overall survival (OS) for all esophageal cancer patients ranges from $15 \%$ to $25 \%$ [3].

The most widely used chemotherapy regimen for metastatic, unresectable esophageal cancer is 5-fluorouracil plus cisplatin, which is based on a small, randomized phase II study that showed higher response rates with 5-fluorouracil plus cisplatin than with cisplatin alone in advanced squamous cell esophageal cancer [4]. Prospective studies of 5-fluorouracil- 
based combination chemotherapy regimens have demonstrated response rates ranging from $20 \%$ to $50 \%$, but longterm outcomes are unsatisfactory.

Irinotecan, a topoisomerase I inhibitor, is active against esophageal cancer, and several studies of single-agent irinotecan for advanced or metastatic esophageal cancer resulted in response rates of $10 \%$ to $20 \%[5,6]$. Due to this limited efficacy of single-agent irinotecan, phase II studies of irinotecan combined with fluorouracil, docetaxel, or cisplatin were performed, and these studies showed activity in firstline and refractory settings [7-12]. A response rate of 57\% was observed in chemotherapy-naïve advanced esophageal cancer patients treated with weekly irinotecan and cisplatin for 4 weeks followed by a 2-week rest period [10]. While this result was encouraging, two-thirds of the patients experienced a dose delay or shortening of treatment because of significant hematologic toxicities. Subsequent studies of combination therapy with modified weekly irinotecan and cisplatin showed conflicting results. Specifically, both demonstrated favourable response rates, but one was associated with reduced hematologic toxicities [13] and the other showed a high hematologic toxicity profile [9]. Irinotecan and cisplatin combination therapy is considered an alternative to 5-fluorouracil plus cisplatin, but most studies of irinotecan and cisplatin have been phase II trials with small sample sizes been conducted in Western populations, which have higher incidences of adenocarcinoma than Asian populations. Therefore, we conducted a multicenter phase II clinical trial to evaluate the efficacy and safety of irinotecan and cisplatin combination chemotherapy for metastatic, unresectable esophageal cancer patients in Korea.

\section{Materials and Methods}

\section{Study design and patients}

We conducted an open-label, single-arm, multicenter, phase II study to evaluate the combination of irinotecan and cisplatin in metastatic, unresectable esophageal cancer. The protocol was approved by the institutional review boards of participating institutions and registered at http://www.cris. nih.go.kr (No. KCT0000670). Written informed consent was obtained from all patients before enrollment, and this trial was conducted according to the principles of the Declaration of Helsinki.

We included patients who met the following eligibility criteria: age of at least 20 years; histologically or cytologically confirmed diagnosis of metastatic or unresectable esophageal cancer (adenocarcinoma or squamous cell carcinoma); cancer that was not curable by radical surgery with curative radiotherapy or concurrent chemoradiotherapy; clear radiologic evidence of disease progression after chemotherapy or local therapy (radiotherapy or surgery); measurable lesions on computed tomography $(\mathrm{CT})$ or magnetic resonance imaging (MRI) as defined by Response Evaluation Criteria in Solid Tumors (RECIST) ver. 1.1; Eastern Cooperative Oncology Group performance status of 0 or 1 ; adequate hematologic function (absolute neutrophils count $[\mathrm{ANC}] \geq 1,500 / \mathrm{mm}^{3}$, platelets $\geq 100,000 / \mathrm{mm}^{3}$, hemoglobin $\geq 9.0 \mathrm{~g} / \mathrm{dL}$ ); adequate renal function (creatinine $\leq 1.5$ times the upper limit of normal); and adequate hepatic function (total bilirubin $\leq 1.5$ times the upper limit of normal and aminotransferase and alanine aminotransferase $\leq 3.0$ times the upper limit of normal); and recovery from adverse events related to previous chemotherapy or local therapy (i.e., grade 0 or 1 at baseline).

\section{Treatment schedule and dosage modifications}

Patients were treated with palliative first-line irinotecan $65 \mathrm{mg} / \mathrm{m}^{2}$ for over 3 hours and cisplatin $30 \mathrm{mg} / \mathrm{m}^{2}$ over 60 minutes intravenously on days 1 and 8 of each 21-day treatment cycle. Chemotherapy was continued for a maximum of six cycles because of the cumulative toxicity of cisplatin.

Dosage modifications were made on the basis of adverse events. The irinotecan dose was reduced to $55 \mathrm{mg} / \mathrm{m}^{2}$ in the event of grade 3 febrile neutropenia, grade 2-4 neutropenia, or grade 2-4 thrombocytopenia. If patients had grade 4 febrile neutropenia, the irinotecan dose was reduced to $45 \mathrm{mg} / \mathrm{m}^{2}$ or permanently discontinued. The irinotecan dose was reduced to $55 \mathrm{mg} / \mathrm{m}^{2}$ in the event of grade 3 or 4 nausea, vomiting, diarrhoea, or oral mucositis. The next cycle was started when hematologic toxicity was resolved to an ANC greater than $1.0 \times 10^{9} / \mathrm{L}$ and platelet count greater than $50 \times 10^{9} / \mathrm{L}$ and when nonhematologic toxicities had recovered to grade 2 or lower. The cisplatin dose was reduced to 20 $\mathrm{mg} / \mathrm{m}^{2}$ in the event of grade 2 peripheral neuropathy or nephrotoxicity (creatinine clearance $[\mathrm{CrCl}], 30$ to $60 \mathrm{~mL} /$ $\mathrm{min})$. Patients who experienced grade 3 or 4 peripheral neuropathy, grade 3 or 4 ototoxicity, grade 3 or 4 cardiac toxicity, or severe nephrotoxicity $(\mathrm{CrCl}<30 \mathrm{~mL} / \mathrm{min})$ were discontinued from the study. Primary prophylactic use of granulocyte-colony stimulating factor (G-CSF) was not permitted, but therapeutic use of G-CSF was allowed after neutropenia was diagnosed. The intention-to-treat (ITT) population included all subjects who received at least one dose of chemotherapy. The per-protocol (PP) population included evaluable patients who completed at least two cycles of chemotherapy and underwent a response assessment with the same modality at baseline and after two cycles of chemotherapy, as well as those who showed confirmed disease progression before the completion of two cycles of chemotherapy. 


\section{Assessment of tumour response and toxicity}

A CT scan or MRI scan was obtained at baseline and repeated every two cycles of chemotherapy to assess tumour response. Patients underwent positron emission tomography-computed tomography (PET-CT) at baseline and after two cycles of chemotherapy to assess initial metabolic response, which was defined as a reduction of at least 30\% in maximum standard uptake values (SUVmax) between baseline and after two cycles of chemotherapy. Tumour response was determined according to RECIST, ver. 1.1 [14]. Toxicity was assessed before every cycle of chemotherapy using the National Cancer Institute Common Terminology Criteria for Adverse Events, ver. 4.0.

\section{Statistical analysis}

The primary endpoint of this study was response rate. Secondary endpoints were progression-free survival (PFS), OS, duration of response, and initial metabolic response rate according to PET-CT. We assumed response rates of $20 \%$ (H0) with 5-fluorouracil plus cisplatin combination chemotherapy and 40\% (H1) with irinotecan and cisplatin combination chemotherapy with a one-sided alpha of $10 \%$ and a power of $80 \%$. Considering a drop-out rate of $10 \%$, a total of 27 patients were required for this study. Survivals were estimated using the Kaplan-Meier method and the logrank test was employed to compare survival between patients with and without initial metabolic response.

\section{Results}

\section{Patient characteristics}

Between September 2012 and March 2015, we enrolled a total of 27 patients from seven centres. Baseline characteristics of the patients are summarized in Table 1. Sixteen patients received planned chemotherapy and four showed confirmed disease progression before completion of two cycles of chemotherapy. Seven patients were excluded from analysis for the following reasons: death before the first tumour assessment $(n=3)$, unacceptable toxicity $(n=2)$, and withdrawal $(\mathrm{n}=2)$. Overall, we were able to assess 20 patients for the PP analysis of efficacy.

\section{Efficacy}

We assessed the efficacy within the PP population $(\mathrm{n}=20)$. No patients achieved a complete response, but six achieved
Table 1. Baseline characteristics

\begin{tabular}{lc} 
Characteristic & No. $(\%)(\mathbf{n}=27)$ \\
Age, median (range, yr) & $61(44-83)$ \\
Sex & \\
Male & $26(96.3)$ \\
Female & $1(3.7)$ \\
ECOG performance status & \\
0 & $1(3.7)$ \\
1 & $26(96.3)$ \\
Location & \\
Cervical & $1(3.7)$ \\
Upper thoracic & $10(37.0)$ \\
Middle thoracic & $6(22.2)$ \\
Lower thoracic & $6(22.2)$ \\
$\quad$ Unknown & $4(14.8)$ \\
Histology & \\
Squamous cell carcinoma & $27(100)$ \\
Adenocarcinoma & 0 \\
Differentiation & \\
Well differentiated & $2(7.4)$ \\
Moderately differentiated & $16(59.3)$ \\
Poorly differentiated & $5(18.5)$ \\
Unknown & $4(14.8)$ \\
Disease status & \\
Initial metastatic & $16(59.3)$ \\
Recurrent after curative treatment & $11(40.7)$ \\
Prior chemotherapy & \\
Yes & $12(44.4)$ \\
No & $15(55.6)$ \\
Prior radiotherapy & \\
Yes & $14(51.9)$ \\
No & $13(48.1)$ \\
\hline &
\end{tabular}

ECOG, Eastern Cooperative Oncology Group.

Table 2. Tumour response

\begin{tabular}{lc} 
Response & No. $(\%)(\mathbf{n}=\mathbf{2 0})$ \\
CR & 0 \\
PR & $6(30.0)$ \\
SD & $9(45.0)$ \\
PD & $5(25.0)$ \\
Response rate (CR+PR) & $6(30.0)$ \\
Disease control rate (CR+PR+SD) & $15(75.0)$ \\
\hline
\end{tabular}

$\mathrm{CR}$, complete response; $\mathrm{PR}$, partial response; $\mathrm{SD}$, stable disease; PD, progressive disease. 


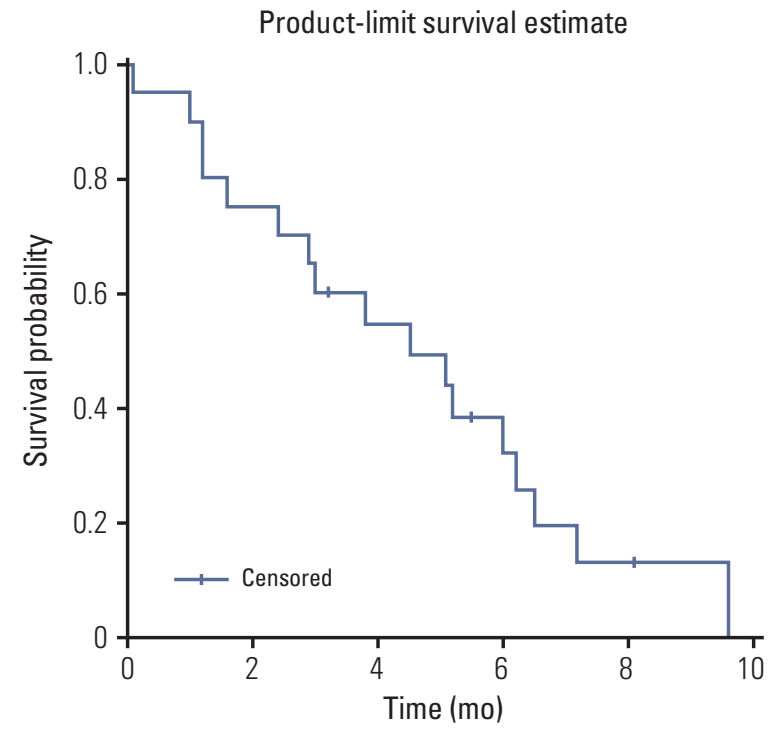

Fig. 1. Progression-free survival for the per protocol population.

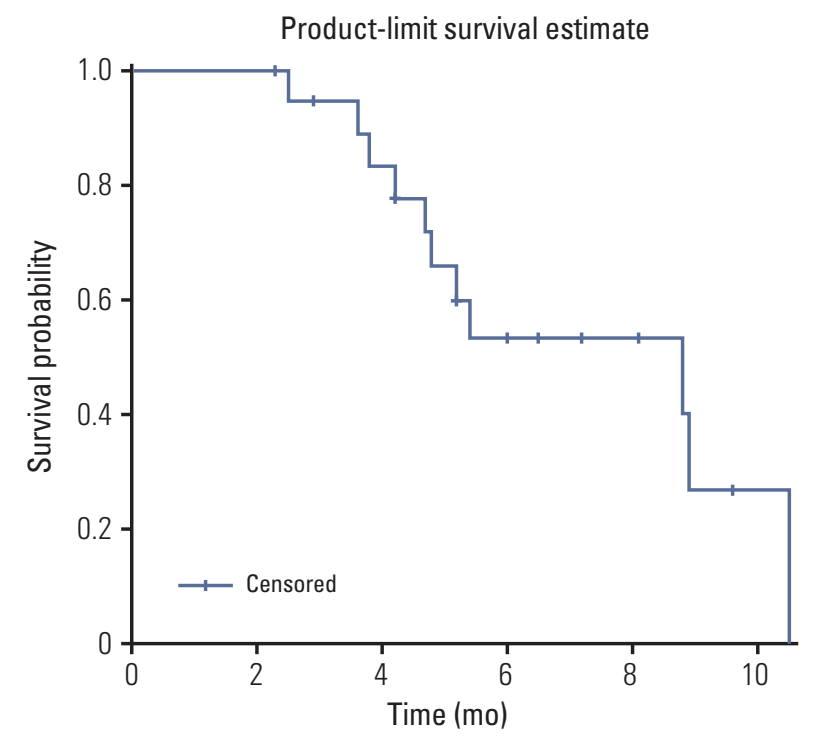

Fig. 2. Overall survival for the per protocol population.

Table 3. Treatment-related toxicity

\begin{tabular}{lcccccc} 
Toxicity & Total $(\mathbf{n = 2 7})$ & Grade $\mathbf{1}$ & Grade 2 & Grade 3 & Grade 4 & Grade 3/4, $\mathbf{n}(\%)$ \\
\hline Anemia & 7 & 0 & 1 & 6 & 0 & $6(22.2)$ \\
Neutropenia & 17 & 0 & 6 & 9 & 2 & $11(40.7)$ \\
Thrombocytopenia & 3 & 1 & 0 & 0 & 2 & $2(7.4)$ \\
Asthenia & 4 & 0 & 0 & 4 & 0 & $4(14.8)$ \\
Fatigue & 4 & 2 & 2 & 0 & 0 & 0 \\
Anorexia & 12 & 6 & 5 & 1 & 0 & $1(3.7)$ \\
Nausea & 11 & 7 & 3 & 0 & 0 & $1(3.7)$ \\
Vomiting & 6 & 4 & 2 & 3 & 0 & 0 \\
Diarrhoea & 16 & 8 & 5 & & 0 & $3(11.1)$ \\
\hline
\end{tabular}

a partial response (PR). The objective response rate was $30.0 \%$ (90\% confidence interval [CI], 13.2 to 46.9) (Table 2) and the median follow-up duration was 10.0 months. The median PFS was 4.5 months (95\% CI, 1.6 to 6.2) (Fig. 1) and the median OS was 8.8 months (95\% CI, 4.7 to 10.5) (Fig. 2). The median duration of response for PR patients was 5.0 months (range, 3.4 to 8.0 months). There were no significant differences in response rate and survivals between the treatment-naïve (i.e., initial metastatic) group and pre-treated (i.e., recurrent after curative treatment) group.

Thirteen patients in the PP group agreed to and underwent a serial PET-CT at baseline and after two cycles of chemotherapy. The mean reduction in SUVmax was $10.6 \%$. Four of the 13 patients $(30.8 \%)$ achieved metabolic response. There were no significant differences in the OS or PFS between patients with and without initial metabolic response $(p=0.51$ and $\mathrm{p}=0.60$, respectively).

\section{Dose administration and toxicity}

The median number of cycles of chemotherapy that patients received was three (range, 1 to 6), and eight patients (29.6\%) completed six cycles of chemotherapy. A total of 93 chemotherapy cycles were administered, of which $15(16.1 \%)$ required dose reduction due to toxicities and 45 cycles $(48.4 \%)$ were delayed. The mean dose intensities of irinotecan and cisplatin were $77.3 \%\left(33.2 \mathrm{mg} / \mathrm{m}^{2} / \mathrm{wk}\right.$ of $43.0 \mathrm{mg} /$ $\left.\mathrm{m}^{2} / \mathrm{wk}\right)$ and $77.1 \%\left(15.4 \mathrm{mg} / \mathrm{m}^{2} / \mathrm{wk}\right.$ of $\left.20.0 \mathrm{mg} / \mathrm{m}^{2} / \mathrm{wk}\right)$ of 
Table 4. Comparison of studies of irinotecan-based chemotherapy for metastatic, unresectable esophageal cancer

\begin{tabular}{|c|c|c|c|c|}
\hline & Present study & Ilson et al. [10] & Ilson et al. [13] & Lee et al. [9] \\
\hline Doses and schedules & $\begin{array}{l}\text { I } 65 \mathrm{mg} / \mathrm{m}^{2} \mathrm{D} 1,8 \\
\text { P } 30 \mathrm{mg} / \mathrm{m}^{2} \mathrm{D} 1,8 \\
\text { Every } 3 \mathrm{wk}\end{array}$ & $\begin{array}{l}\mathrm{I} 65 \mathrm{mg} / \mathrm{m}^{2} \\
\mathrm{D} 1,8,15,22 \\
\mathrm{P} 30 \mathrm{mg} / \mathrm{m}^{2} \\
\mathrm{D} 1,8,15,22 \\
\text { Every } 6 \mathrm{wk}\end{array}$ & $\begin{array}{l}\text { I } 65 \mathrm{mg} / \mathrm{m}^{2} \\
\text { D1, } 8 \\
\text { P } 30 \mathrm{mg} / \mathrm{m}^{2} \\
\text { D1, } 8 \\
\text { Every } 3 \mathrm{wk}\end{array}$ & $\begin{array}{l}\text { I } 65 \mathrm{mg} / \mathrm{m}^{2} \\
\text { D1, } 8 \\
\text { P } 30 \mathrm{mg} / \mathrm{m}^{2} \\
\text { D1, } 8 \\
\text { Every } 3 \mathrm{wk}\end{array}$ \\
\hline No. of enrolled patients & 27 & 38 & 39 & 32 \\
\hline Disease status & $\begin{array}{l}\text { Metastatic, } \\
\text { unresectable, } \\
\text { chemo-naïve }\end{array}$ & $\begin{array}{l}\text { Metastatic, } \\
\text { unresectable, } \\
\text { chemo-naïve }\end{array}$ & $\begin{array}{l}\text { Metastatic, } \\
\text { unresectable, } \\
\text { chemo-naïve }\end{array}$ & $\begin{array}{l}\text { Metastatic, } \\
\text { unresectable, } \\
\text { chemo-naïve }\end{array}$ \\
\hline Squamous cancer & $27(100)$ & $23(65.7)$ & $29(74.4)$ & $32(100)$ \\
\hline Adenocarcinoma & 0 & $12(34.3)$ & $10(25.6)$ & 0 \\
\hline Response rate & $6 / 20(30)$ & $20 / 35(57)$ & $10 / 28(36)$ & $10 / 32(31)$ \\
\hline Median PFS (mo) & 4.5 & - & - & 4.4 \\
\hline Median OS (mo) & 8.8 & 14.6 & - & 9.6 \\
\hline Grade $3 / 4$ neutropenia & $11 / 27(41)$ & $16 / 35(46)$ & $8 / 36(22)$ & $16 / 32(50)$ \\
\hline Grade 3/4 diarrhea & $3 / 27(11)$ & $4 / 35(11)$ & $7 / 36(19)$ & 4/32 (12) \\
\hline
\end{tabular}

Values are presented as number (\%). I, irinotecan; P, cisplatin; PFS, progression-free survival; OS, overall survival.

the planned doses, respectively.

Toxicity was assessed by ITT for all 27 patients who received at least one dose of chemotherapy. Treatmentrelated toxicities $(\geq 10 \%)$ are summarized in Table 3 . The most common grade $3 / 4$ hematologic toxicity was neutropenia $(40.7 \%)$. Two patients $(7.4 \%)$ experienced febrile neutropenia. The most common grade $3 / 4$ non-hematologic toxicities were asthenia $(14.8 \%)$ and diarrhoea $(11.1 \%)$. Three patients required hospitalization because of diarrhoea. There was one treatment-related death caused by pneumonia and septic shock.

\section{Discussion}

This multicenter phase II study of irinotecan and cisplatin combination chemotherapy for metastatic, unresectable esophageal cancer demonstrated a response rate of $30.0 \%$ with a median PFS of 4.5 months and a median OS of 8.8 months. Results from similar studies of irinotecan-based chemotherapy are summarized in Table 4 . The efficacy observed in this study was comparable to that reported in a previous phase II study of weekly irinotecan and cisplatin combination in a Korean population [9], as well as other studies of different combination chemotherapy regimens including 5-fluorouracil plus cisplatin. Specifically, relative dose intensities of irinotecan and cisplatin and the toxicity profiles observed in our study were similar to those observed in the phase II study in Korea.

When compared with a previous phase II study that used a consecutive-weeks treatment schedule [10], this study required fewer dose reductions $(16.1 \%$ vs. $20.0 \%)$ and fewer dose delays ( $48.4 \%$ vs. $65.7 \%$ ). Nevertheless, we observed a relatively high incidence of grade $3 / 4$ neutropenia of $40.7 \%$ and neutropenic fever in $7.4 \%$ of patients. However, all cases of grade $3 / 4$ neutropenia and febrile neutropenia were resolved after treatment with appropriate antibiotics and G-CSF. Although dose reduction at the first cycle of chemotherapy and prophylactic use of G-CSF were not allowed in this study, both could be considered in patients with risk factors such as old age, multiple comorbidities, or poor nutritional status to alleviate the risk of grade $3 / 4$ neutropenia. The incidence of grade $3 / 4$ treatment-related diarrhoea in this study was $11.1 \%$, which is similar to the incidences reported in other studies of irinotecan and cisplatin regimens $[9,10]$.

The predictive and prognostic roles of metabolic response according to PET-CT in esophageal cancer have been actively investigated, especially in patients receiving neoadjuvant treatment [15-19], but the results have been inconsistent. In this study, initial metabolic response after two cycles of chemotherapy did not predict outcomes, although we did observe a mean reduction in SUVmax of $10.6 \%$. Our study was too small to clearly identify the role of PET-CT in metastatic, unresectable esophageal cancer because of the lack of statistical power. 
Although the small sample size is a limitation to this study, our findings are clinically meaningful. Irinotecan and cisplatin combination is relatively well tolerated as an outpatient-based regimen and would be an effective treatment option for patients who experience early recurrence after fluorouracil-based chemoradiation. There is increasing evidence that efficacy and toxicity profiles of irinotecan may be influenced by inter-individual and interethnic variabilities in genetic polymorphisms [20]. Few studies have investigated irinotecan-based chemotherapy for Korean esophageal cancer patients, so it is important to validate the regimen and identify more optimal doses and schedules.

\section{Conclusion}

Irinotecan and cisplatin combination chemotherapy has modest anti-tumour activity and manageable toxicity in metastatic, unresectable esophageal cancer.

\section{Conflicts of Interest}

Irinotecan was supported by Hanmi Pharm. Co, Ltd.

\section{Acknowledgments}

This research was supported by a grant (12172MFDS231) from the Ministry of Food and Drug Safety in 2012. The research was supported by the Korean Cancer Study Group (KCSG HN13-06).

\section{Author Details}

${ }^{1}$ Department of Internal Medicine, Seoul National University Hospital, Seoul National University College of Medicine, Seoul, ${ }^{2}$ Department of Internal Medicine, Institute of Health Sciences, Gyeongsang National University Hospital, Gyeongsang National University School of Medicine, Jinju, ${ }^{3}$ Department of Internal Medicine, SMG-SNU Boramae Medical Center, Seoul, ${ }^{4}$ Department of Hematology-Oncology, Inje University Haeundae Paik Hospital, Busan, ${ }^{5}$ Department of Internal Medicine, Kosin University Gospel Hospital, Busan, ${ }^{6}$ Department of Internal Medicine, Yeungnam University College of Medicine, Daegu, ${ }^{7}$ Department of Hematology-Oncology, Keimyung University School of Medicine, Daegu, ${ }^{8}$ Department of Internal Medicine, Chungnam National University College of Medicine, Daejeon, ${ }^{9}$ Department of Internal Medicine, Seoul National University Bundang Hospital, Seongnam, Korea

\section{References}

1. Torre LA, Bray F, Siegel RL, Ferlay J, Lortet-Tieulent J, Jemal A. Global cancer statistics, 2012. CA Cancer J Clin. 2015;65:87108.

2. Jung KW, Won YJ, Kong HJ, Oh CM, Cho H, Lee DH, et al. Cancer statistics in Korea: incidence, mortality, survival, and prevalence in 2012. Cancer Res Treat. 2015;47:127-41.

3. Pennathur A, Gibson MK, Jobe BA, Luketich JD. Oesophageal carcinoma. Lancet. 2013;381:400-12.

4. Bleiberg H, Conroy T, Paillot B, Lacave AJ, Blijham G, Jacob $\mathrm{JH}$, et al. Randomised phase II study of cisplatin and 5-fluorouracil (5-FU) versus cisplatin alone in advanced squamous cell oesophageal cancer. Eur J Cancer. 1997;33:1216-20.

5. Muhr-Wilkenshoff F, Hinkelbein W, Ohnesorge I, Wolf KJ, Riecken EO, Zeitz M, et al. A pilot study of irinotecan (CPT11) as single-agent therapy in patients with locally advanced or metastatic esophageal carcinoma. Int J Colorectal Dis. 2003;18:330-4.

6. Enzinger PC, Kulke MH, Clark JW, Ryan DP, Kim H, Earle $\mathrm{CC}$, et al. A phase II trial of irinotecan in patients with previ- ously untreated advanced esophageal and gastric adenocarcinoma. Dig Dis Sci. 2005;50:2218-23.

7. Wolff K, Wein A, Reulbach U, Mannlein G, Bruckl V, Meier C, et al. Weekly high-dose 5-fluorouracil as a 24-h infusion and sodium folinic acid (AIO regimen) plus irinotecan in patients with locally advanced nonresectable and metastatic adenocarcinoma or squamous cell carcinoma of the oesophagus: a phase II trial. Anticancer Drugs. 2009;20:165-73.

8. Enzinger PC, Ryan DP, Clark JW, Muzikansky A, Earle CC, Kulke $\mathrm{MH}$, et al. Weekly docetaxel, cisplatin, and irinotecan (TPC): results of a multicenter phase II trial in patients with metastatic esophagogastric cancer. Ann Oncol. 2009;20:475-80.

9. Lee DH, Kim HT, Han JY, Lee SY, Yoon SJ, Kim HY, et al. A phase II trial of modified weekly irinotecan and cisplatin for chemotherapy-naive patients with metastatic or recurrent squamous cell carcinoma of the esophagus. Cancer Chemother Pharmacol. 2008;61:83-8.

10. Ilson DH, Saltz L, Enzinger P, Huang Y, Kornblith A, Gollub $\mathrm{M}$, et al. Phase II trial of weekly irinotecan plus cisplatin in 
advanced esophageal cancer. J Clin Oncol. 1999;17:3270-5.

11. Assersohn L, Brown G, Cunningham D, Ward C, Oates J, Waters JS, et al. Phase II study of irinotecan and 5-fluorouracil/leucovorin in patients with primary refractory or relapsed advanced oesophageal and gastric carcinoma. Ann Oncol. 2004;15:64-9.

12. Burtness B, Gibson M, Egleston B, Mehra R, Thomas L, Sipples $\mathrm{R}$, et al. Phase II trial of docetaxel-irinotecan combination in advanced esophageal cancer. Ann Oncol. 2009;20:1242-8.

13. Ilson DH. Phase II trial of weekly irinotecan/cisplatin in advanced esophageal cancer. Oncology (Williston Park). 2004;18(14 Suppl 14):22-5.

14. Eisenhauer EA, Therasse P, Bogaerts J, Schwartz LH, Sargent D, Ford R, et al. New response evaluation criteria in solid tumours: revised RECIST guideline (version 1.1). Eur J Cancer. 2009;45:228-47.

15. Elliott JA, O'Farrell NJ, King S, Halpenny D, Malik V, Muldoon $\mathrm{C}$, et al. Value of CT-PET after neoadjuvant chemoradiation in the prediction of histological tumour regression, nodal status and survival in oesophageal adenocarcinoma. Br J Surg. 2014;101:1702-11.
16. Tamandl D, Gore RM, Fueger B, Kinsperger P, Hejna M, Paireder $\mathrm{M}$, et al. Change in volume parameters induced by neoadjuvant chemotherapy provide accurate prediction of overall survival after resection in patients with oesophageal cancer. Eur Radiol. 2016;26:311-21.

17. Pan L, Gu P, Huang G, Xue H, Wu S. Prognostic significance of SUV on PET/CT in patients with esophageal cancer: a systematic review and meta-analysis. Eur J Gastroenterol Hepatol. 2009;21:1008-15.

18. Gillies RS, Middleton MR, Blesing C, Patel K, Warner N, Marshall RE, et al. Metabolic response at repeat PET/CT predicts pathological response to neoadjuvant chemotherapy in oesophageal cancer. Eur Radiol. 2012;22:2035-43.

19. Kukar M, Alnaji RM, Jabi F, Platz TA, Attwood K, Nava H, et al. Role of repeat $18 \mathrm{~F}$-fluorodeoxyglucose positron emission tomography examination in predicting pathologic response following neoadjuvant chemoradiotherapy for esophageal adenocarcinoma. JAMA Surg. 2015;150:555-62.

20. Shimoyama S. Pharmacogenetics of irinotecan: An ethnicitybased prediction of irinotecan adverse events. World J Gastrointest Surg. 2010;2:14-21. 